\title{
Identification of myopia-associated WNT7B polymorphisms provides insights into the mechanism underlying the development of myopia
}

\author{
Masahiro Miyake1,2, Kenji Yamashiro1, Yasuharu Tabara², Kenji Suda1', Satoshi Morooka1, Hideo Nakanishi',
} Chiea-Chuen Khor 3,4,5,6, Peng Chen ${ }^{3}$, Fan Qiao ${ }^{3}$, Isao Nakata1,2, Yumiko Akagi-Kurashige ${ }^{1,2}$, Norimoto Gotoh², Akitaka Tsujikawa', Akira Meguro ${ }^{7}$, Sentaro Kusuhara ${ }^{8}$, Ozen Polasek ${ }^{9}$, Caroline Hayward ${ }^{10}$, Alan F. Wright ${ }^{10}$, Harry Campbell ${ }^{11}$, Andrea J. Richardson ${ }^{12}$, Maria Schache ${ }^{12}$, Masaki Takeuchi ${ }^{7,13}$, David A. Mackey ${ }^{12,14}$, Alex W. Hewitt ${ }^{12}$, Gabriel Cuellar ${ }^{15}$, Yi Shi ${ }^{16}$, Luling Huang ${ }^{16}$, Zhenglin Yang ${ }^{16,17,18}$, Kim Hung Leung ${ }^{19}$, Patrick Y.P. Kao ${ }^{20}$, Maurice K.H. Yap ${ }^{20}$, Shea Ping Yip ${ }^{19}$, Muka Moriyama ${ }^{21}$, Kyoko Ohno-Matsui ${ }^{21}$, Nobuhisa Mizuki ${ }^{7}$, Stuart MacGregor ${ }^{15}$, Veronique Vitart ${ }^{10}$, Tin Aung ${ }^{4,22}$, Seang-Mei Saw ${ }^{3,4,22}$, E-Shyong Tai 3,23,24, Tien Yin Wong 4,21,22, Ching-Yu Cheng 4,22,24, Paul N. Baird ${ }^{12}$, Ryo Yamada 2 , Fumihiko Matsuda ${ }^{2}$, Nagahama Study Group ${ }^{\star} \&$ Nagahisa Yoshimura ${ }^{1}$

Myopia can cause severe visual impairment. Here, we report a two-stage genome-wide association study for three myopia-related traits in 9,804 Japanese individuals, which was extended with trans-ethnic replication in 2,674 Chinese and 2,690 Caucasian individuals. We identify $W N T 7 B$ as a novel susceptibility gene for axial length ( $r$ s10453441, $P_{\text {meta }}=3.9$ $\left.\times 10^{-13}\right)$ and corneal curvature $\left(P_{\text {meta }}=2.9 \times 10^{-40}\right)$ and confirm the previously reported association between GJD2 and myopia. WNT7B significantly associates with extreme myopia in a case-control study with 1,478 Asian patients and 4,689 controls (odds ratio $(O R)_{\text {meta }}=1.13, P_{\text {meta }}=0.011$. We also find in a mouse model of myopia downregulation of WNT7B expression in the cornea and upregulation in the retina, suggesting its possible role in the development of myopia.

\footnotetext{
${ }^{1}$ Department of Ophthalmology and Visual Science, Kyoto University Graduate School of Medicine, Kyoto 6068507, Japan. ${ }^{2}$ Center for Genomic Medicine, Kyoto University Graduate School of Medicine, Kyoto 6068503, Japan. ${ }^{3}$ Saw Swee Hock School of Public Health, National University of Singapore and National University Health System, Singapore 117597, Singapore. ${ }^{4}$ Department of Ophthalmology, National University of Singapore and National University Health System, Singapore 119228, Singapore. ${ }^{5}$ Department of Pediatrics, National University of Singapore, Singapore 119077, Singapore. ${ }^{6}$ Division of Human Genetics, Genome Institute of Singapore, Singapore 138672, Singapore. ${ }^{7}$ Department of Ophthalmology and Visual Science, Yokohama City University Graduate School of Medicine, Yokohama 2360027, Japan. ${ }^{8}$ Division of Ophthalmology, Department of Surgery, Kobe University Graduate School of Medicine, Kobe 6570013, Japan. ${ }^{9}$ Faculty of Medicine, University of Split, Split 21000, Croatia. ${ }^{10}$ Medical Research Council Human Genetics Unit, Institute of Genetics and Molecular Medicine, University of Edinburgh, Edinburgh EH8 9YL, UK. ${ }^{11}$ Centre for Population Health Sciences, Medical School, Teviot, Edinburgh EH8 9AG, UK. ${ }^{12}$ Centre for Eye Research Australia (CERA), University of Melbourne, Royal Victorian Eye and Ear Hospital, Melbourne, Victoria 3002, Australia. ${ }^{13}$ Inflammatory Disease Section, Medical Genetics Branch, National Human Genome Research Institute, National Institutes of Health, Bethesda, Maryland 20892, USA. ${ }^{14}$ Centre for Ophthalmology and Visual Science, Lions Eye Institute, University of Western Australia, Perth, Western Australia 6009, Australia. ${ }^{15}$ Statistical Genetics, QIMR Berghofer Medical Research Institute, Brisbane, Queensland 4006, Australia. ${ }^{16}$ Sichuan Provincial Key Laboratory for Human Disease Gene Study, Hospital of the University of Electronic Science and Technology of China and Sichuan Provincial People's Hospital, Chengdu, Sichuan 610072, China. ${ }^{17}$ School of Medicine, University of Electronic Science and Technology of China and Sichuan Provincial People's Hospital, Chengdu, Sichuan 610072, China. ${ }^{18}$ Sichuan Translational Medicine Hospital, Chinese Academy of Sciences, Chengdu, Sichuan 610072, China. ${ }^{19}$ Department of Health Technology and Informatics, The Hong Kong Polytechnic University, Hong Kong SAR 99907, China. ${ }^{20}$ Centre for Myopia Research, School of Optometry, The Hong Kong Polytechnic University, Hong Kong SAR 99907, China. ${ }^{21}$ Department of Ophthalmology and Visual Science, Tokyo Medical and Dental University, Tokyo 1130034, Japan. ${ }^{22}$ Singapore Eye Research Institute, Singapore National Eye Centre, Singapore 168751, Singapore.

${ }^{23}$ Department of Medicine, National University of Singapore and National University Health System, Singapore 119228, Singapore. ${ }^{24}$ Duke-NUS Graduate Medical School, Singapore 169857, Singapore. Correspondence and requests for materials should be addressed to K.Y. (email: yamashro@kuhp.kyoto-u.ac.jp).

*Lists of members and their affiliations appear at the end of the paper.
} 
. igh myopia increases the risk of other pathological ocular complications $^{1}$, owing to which myopia is emerging as a major public health concern in many parts of the world. The prevalence of high myopia and associated visual complications is increasing in many Asian and Caucasian populations, and this trend is particularly notable in urbanized areas $^{2-4}$. The World Health Organization recognizes myopia as a major cause of visual impairment ${ }^{5}$.

Emmetropia, the absence of refractive error, is determined by the precise coordination of ocular parameters, such as axial length, corneal curvature, lens thickness and anterior chamber depth. Disruption of this coordination causes refractive errors, such as myopia and hyperopia ${ }^{6,7}$. Of these components, axial length plays a major role in refraction; therefore, in clinical settings, the degree of myopia is predominantly determined by axial length ${ }^{8-10}$. A recent large-scale, genome-wide meta-analysis by the Consortium for Refractive Error and Myopia (CREAM) reported that 23 of 29 loci associated with refraction were also associated with axial length ${ }^{11,12}$. However, genes associated with axial length are not necessarily associated with refractive error ${ }^{12}$. This can be explained on the basis of the compensatory effects of other ocular biometric components, such as corneal curvature that influence the effect of an increased axial length through a flatter cornea ${ }^{6}$. Indeed, in genome-wide association studies (GWASs), some genes found to be associated with corneal curvature were also recently reported to be associated with axial length, but not with refractive error ${ }^{7,13}$.

On the other hand, the retina is thought to play a crucial role in the development of myopia ${ }^{14}$. For example, it is well known that central hyperopic defocus can cause myopia in chicks ${ }^{15}$ and mice models ${ }^{16}$. In addition, experiments conducted by Smith et al. ${ }^{17-19}$ have shown that peripheral hyperopic defocus can cause myopic shift in monkeys. This is a herald of 'peripheral defocus theory' that implicates defocus at the peripheral retina as the trigger for myopia. These reports suggest that the retina can perceive the defocus and evoke signals to accelerate the progression of myopia. This hypothesis is supported by animal experiments that show amacrine cells and their transcription factor, ZENK, have important roles in the perception of retinal defocus ${ }^{20,21}$. Nevertheless, the mechanisms by which these signals are transduced are not yet known.

In the current study, we conduct a two-stage GWAS on three myopia-related traits-axial length, spherical error and corneal curvature-within the Nagahama Prospective Genome Cohort for Comprehensive Human Bioscience (the Nagahama Study), which involves 9,804 healthy Japanese individuals. The analysis of an additional 2,674 Chinese individuals across two independent cohorts and 2,690 Caucasian individuals across five independent cohorts confirms the trans-ethnic genetic associations. In addition, we evaluate the associations with extreme myopia in Asian individuals.

\section{Results}

Two-stage GWAS within the Nagahama study. The cohort demographics used in this study are shown in Supplementary Table 1. After stringent quality control, a total of 1,773,334 single nucleotide polymorphisms (SNPs) were included in the analyses of the Nagahama cohort. Axial length, spherical equivalent and corneal curvature were used as the dependent variables for three genome-wide quantitative trait loci (QTL) analyses. We included age, sex and height as covariates, but did not include principal components in these analyses. However, inflation factors $\left(\lambda_{\mathrm{GC}}\right)$ of 1.031 (axial length), 1.024 (spherical equivalent) and 1.016 (corneal curvature) indicated excellent control of population substructure. The QQ plots are shown in the Supplementary Fig. 1.
During the first stage, we identified five loci that showed a suggestive association with a $P$ value $<1.0 \times 10^{-6}$ by linear regression; one of these SNPs was WNT7B rs200329677, which exceeded genome-wide significance with a $P$ value of $1.13 \times 10^{-9}$ in the association with corneal curvature (Fig. 1 and Table 1). In the following replication stage, we further genotyped 3,460 Japanese individuals for three SNPs that showed suggestive association with a $P$ value $<1.0 \times 10^{-6}$ and had a minor allele frequency (MAF) of at least $5 \%$ in the discovery stage. We investigated the associations of each of the three traits with each SNP and evaluated the results of the first and second stage in a meta-analysis (Table 2). This analysis revealed associations with two traits for the two SNPs in GJD2 and WNT7B. First, we successfully replicated the previously reported association between GJD2 rs11073058 and axial length $\left(P_{\text {combined }}=2.4 \times\right.$ $10^{-8}$; inverse variance meta-analysis of discovery and replication

\section{a}

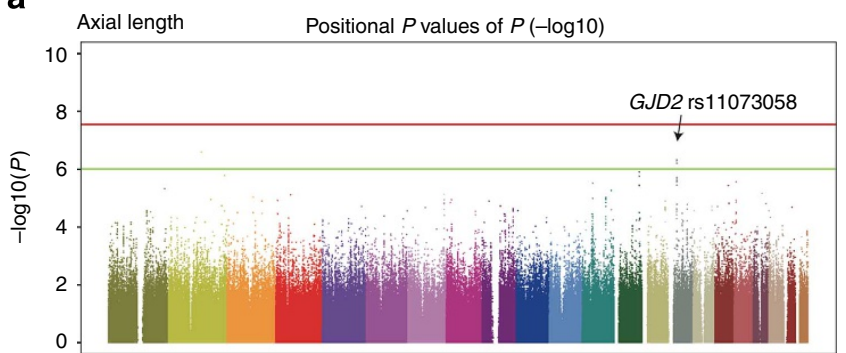

b

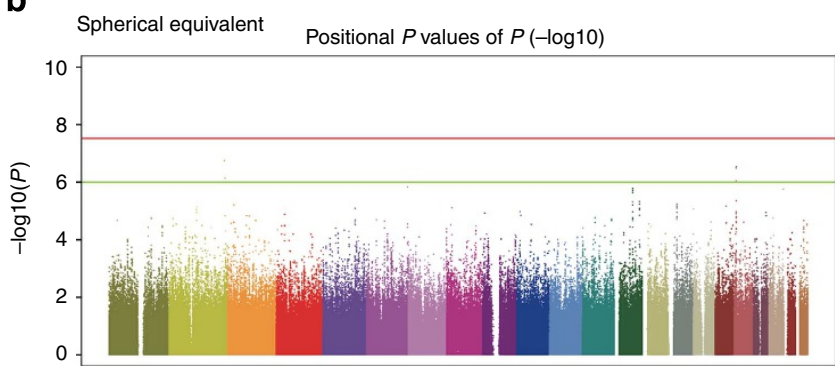

C

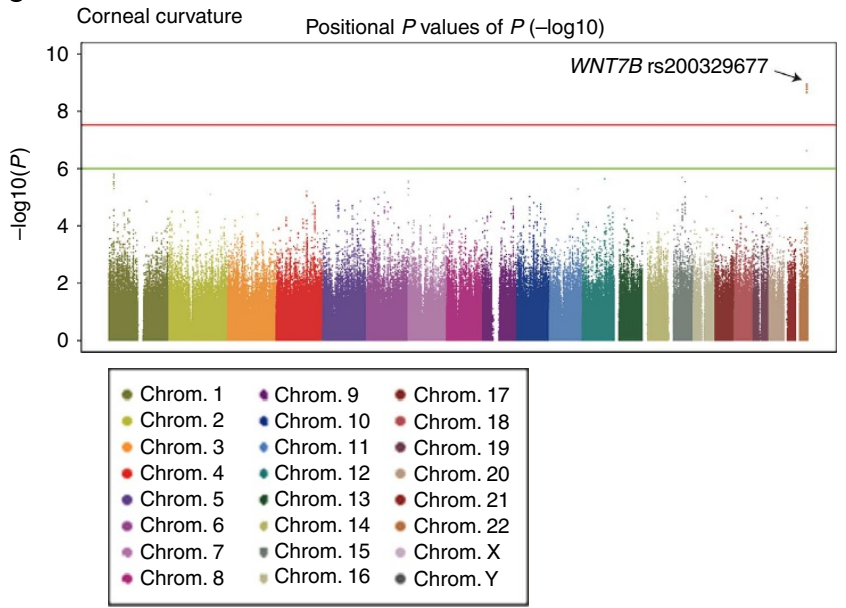

Figure 1 | Manhattan plots of the first stage GWAS for three myopiarelated traits. Each plot shows $-\log _{10}$-transformed $P$ values for all SNPs. The upper horizontal line represents the genome-wide significance threshold of $P<2.82 \times 10^{-8}$, and the lower line represents the suggestive threshold of $P<1.00 \times 10^{-6}$. Nine SNPs from five genes surpassed the suggestive $P$ value, including previously reported GJD2 $\left(P=4.72 \times 10^{-7}\right.$ by linear regression using 2,991 individuals) for axial length and the novel genome-wide hit WNT7B for corneal curvature $\left(P_{\text {top }}=1.13 \times 10^{-9}\right.$ by linear regression using 2,747 ) 
Table 1 | Results of the discovery stage.

\begin{tabular}{|c|c|c|c|c|c|c|c|c|c|c|c|}
\hline & Nearby genes & & rs number & CHR & Position & $n$ & $\begin{array}{c}\text { Effect } \\
\text { allele }\end{array}$ & $\beta$ & $\begin{array}{c}\text { Standard } \\
\text { error }\end{array}$ & MAF & $P$ value \\
\hline Axial length & THSD7B & $\begin{array}{l}\text { Not in } \\
\text { gene }\end{array}$ & rs78534307 & 2 & $1,368,233,336$ & 2,991 & G & 0.83 & 0.16 & 0.01 & $2.52 \times 10^{-7}$ \\
\hline \multirow[t]{2}{*}{$\begin{array}{l}\text { Spherical } \\
\text { equivalent }\end{array}$} & LOC101928675 & $\begin{array}{l}\text { Not in } \\
\text { gene }\end{array}$ & rs6732520 & 2 & $228,821,808$ & 2,730 & C & -1.85 & 0.35 & 0.01 & $1.73 \times 10^{-7}$ \\
\hline & RPL6P27 & $\begin{array}{l}\text { Not in } \\
\text { gene }\end{array}$ & rs4798444 & 18 & $6,455,641$ & 2,730 & A & 0.36 & 0.07 & 0.50 & $2.86 \times 10^{-7}$ \\
\hline
\end{tabular}

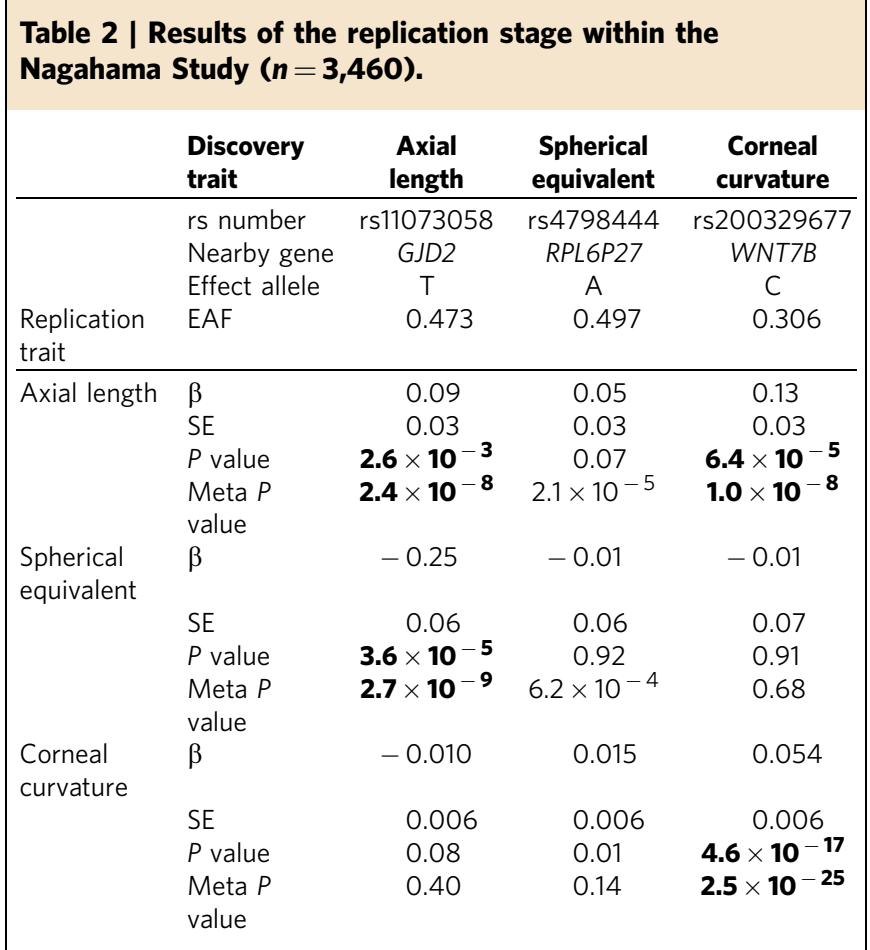

Associations that reached genome-wide significance in the meta-analysis are described in bold. $E A F$, effect allele frequency.

$P$ values derived using linear regression.

results from Japanese cohort) as well as spherical equivalent $\left(P_{\text {combined }}=2.7 \times 10^{-9}\right)$. We did not observe any association between GJD2 rs11073058 and corneal curvature. Second, we confirmed the novel associations of WNT7B rs200329677 with corneal curvature $\left(P_{\text {combined }}=2.5 \times 10^{-25}\right)$ and axial length $\left(P_{\text {combined }}=1.0 \times 10^{-8}\right)$. This SNP did not show association with the spherical equivalent.

Replication in other ethnicities and meta-analysis. Of the current commercially available DNA microarrays, the WNT7B rs200329677 was included only on the Illumina HumanOmni 2.5 and $5 \mathrm{M}$ chips. This SNP is not available in the 1000 Genomes Project or in the HapMap Project; thus, imputation to these reference genomes did not allow us to directly assess rs200329677 for association. Association with a trait was sought with rs10453441, which was in very high linkage disequilibrium with rs200329677 $\left(R^{2}=0.967\right.$ in the Nagahama Study data set), and strong evidence was found for association to corneal curvature $\left(P_{\text {discovery }}=2.16 \times 10^{-9}\right.$ and $P_{\text {replication }}=4.6 \times 10^{-17}$ in the Nagahama data sets).

We conducted replication of both SNPs in an additional 2,674 Chinese individuals across two independent collections, as well as across 2,835 Caucasian individuals across five collections (Supplementary Methods). The SNP rs10453441 showed significant evidence of replication (Table 3 ) for axial length and corneal curvature both in Asian $\left(P_{\mathrm{AL}}=7.1 \times 10^{-6}\right.$, $P_{\mathrm{CC}}=3.0 \times 10^{-14}$ ) and, to a lesser extent, in Caucasian $\left(P_{\mathrm{AL}}=2.0 \times 10^{-3}, P_{\mathrm{CC}}=4.1 \times 10^{-2}\right)$ groups, with no heterogeneity across the two collections of Asian individuals $\left(I_{\mathrm{AL}}^{2}=0\right.$, $\left.I^{2} \mathrm{CC}=0\right)$ and moderate-to-high heterogeneity across the five collections of Caucasian individuals $\left(I_{\mathrm{AL}}^{2}=0.38, I_{\mathrm{CC}}^{2}=0.89\right)$. Meta-analysis of the data from all the Asian collections showed genome-wide significant associations with both axial length $\left(P_{\text {meta-Asian }}=1.20 \times 10^{-9}, \beta=0.129 \mathrm{~mm}\right)$ and corneal curvature $\left(P_{\text {meta-Asian }}=9.00 \times 10^{-39}, \beta=0.053 \mathrm{~mm}\right)$, with no evidence of heterogeneity.

Finally, meta-analysis of the Asian and Caucasian data also revealed strong associations between $W N T 7 B$ rs 10453441 and axial length $\left(P_{\text {meta-ALL }}=3.9 \times 10^{-9}, \beta=0.121 \pm 0.017 \mathrm{~mm}\right)$, as well as corneal curvature $\left(P_{\text {meta-ALL }}=2.7 \times 10^{-40}\right.$, $\beta=0.051 \pm 0.004 \mathrm{~mm})$.

Only in the Genes in Myopia cohort ${ }^{22}$ could we obtain Caucasian genotypes for both rs10453441 and rs200329677, so that we could assess the linkage disequilibrium (LD) between these two SNPs in Caucasians. They were in moderate LD $\left(R^{2}=0.499, D^{\prime}=0.943\right)$.

Possible interactive effect of $W N T 7 B$ and GJD2. GJD2 is an established susceptibility gene for increased axial length and myopic refraction ${ }^{11,23}$. In the current study, these associations with GJD2 were replicated in our independent Japanese sample collections. To further our understanding of the role of GJD2 and $W N T 7 B$, we investigated the potential interactive effect of these two genes on myopia susceptibility by the directly genotyped cohorts: Nagahama replication samples, SCES, SP2, Raine, Croatia-split, ORCADES and Genes in Myopia. Figure 2 shows the effect of the GJD2 rs11073058 T allele on spherical equivalent, stratifying by the genotypes of WNT7B rs10453441. As described in Fig. 2, in the Asian populations, the effects of GJD2 rs 11073058 on spherical equivalent were the highest when individuals were homozygous (AA genotype) at WNT7B rs10453441 $(\beta=-0.54 \mathrm{D} ; 95 \% \mathrm{CI}:-0.82$ to $-0.27 \mathrm{D})$ compared with that in the individuals with the AG genotype $(\beta=-0.24 \mathrm{D}$; 
Table 3 | Replication of the association of WNT7B SNPs with three traits.

\begin{tabular}{|c|c|c|c|c|c|c|c|c|c|c|c|c|c|c|}
\hline \multirow[t]{2}{*}{ SNP } & \multirow{2}{*}{$\begin{array}{l}\text { Effect } \\
\text { allele }\end{array}$} & \multirow[t]{2}{*}{ Cohort $^{\star}$} & \multirow[t]{2}{*}{ Ethnicity } & \multirow[t]{2}{*}{$n$} & \multirow[t]{2}{*}{ EAF } & \multicolumn{3}{|c|}{ Axial length } & \multicolumn{3}{|c|}{ Corneal curvature } & \multicolumn{3}{|c|}{ Spherical equivalent } \\
\hline & & & & & & Beta & SE & $P$ value & Beta & SE & $P$ value & Beta & SE & $P$ value \\
\hline \multirow[t]{3}{*}{ rs200329677 } & \multirow[t]{3}{*}{ C } & Nagahama & Japanese & 3,306 & 0.31 & 0.13 & 0.03 & $6.4 \times 10^{-5}$ & 0.054 & 0.006 & $4.6 \times 10^{-17}$ & -0.08 & 0.07 & 0.91 \\
\hline & & SCES & Chinese & 1,771 & 0.30 & 0.19 & 0.05 & $1.2 \times 10^{-4}$ & 0.060 & 0.009 & $207 \times 10^{-10}$ & -0.03 & 0.10 & 0.80 \\
\hline & & GEM & Caucasian & 534 & 0.41 & 0.08 & 0.10 & 0.42 & 0.008 & 0.066 & 0.91 & -0.24 & 0.19 & 0.21 \\
\hline \multirow[t]{12}{*}{ rs10453441 } & \multirow[t]{12}{*}{ A } & Nagahama & Japanese & 3,460 & 0.31 & 0.11 & 0.03 & $7.0 \times 10^{-4}$ & 0.055 & 0.006 & $2.5 \times 10^{-18}$ & 0.04 & 0.06 & 0.52 \\
\hline & & SCES & Chinese & 1,923 & 0.33 & 0.14 & 0.05 & $3.1 \times 10^{-3}$ & 0.054 & 0.009 & $1.2 \times 10^{-9}$ & 0.03 & 0.10 & 0.74 \\
\hline & & $\mathrm{SP} 2^{\dagger}$ & Chinese & 851 & 0.33 & - & - & - & 0.066 & 0.014 & $2.3 \times 10^{-6}$ & 0.05 & 0.14 & 0.74 \\
\hline & & $\begin{array}{l}\text { Meta } \\
\text { Asian } \\
\text { replication }\end{array}$ & - & $\begin{array}{l}5,383 \\
6,234\end{array}$ & - & 0.12 & 0.03 & $7.1 \times 10^{-6}$ & 0.058 & 0.008 & $3.0 \times 10^{-14}$ & 0.04 & 0.05 & 0.43 \\
\hline & & $\begin{array}{l}\text { Meta } \\
\text { Asian }\end{array}$ & - & $\begin{array}{c}8,374 \\
8,981\end{array}$ & - & 0.13 & 0.02 & $1.2 \times 10^{-9}$ & 0.053 & 0.004 & $9.0 \times 10^{-39}$ & 0.02 & 0.04 & 0.58 \\
\hline & & ORCADES $\ddagger$ & Caucasian & 677 & 0.55 & 0.19 & 0.05 & $7.4 \times 10^{-4}$ & - & - & - & 0.07 & 0.11 & 0.54 \\
\hline & & GEM & Caucasian & 551 & 0.56 & 0.05 & 0.10 & 0.60 & 0.084 & 0.064 & 0.19 & 0.08 & 0.06 & 0.19 \\
\hline & & $\begin{array}{l}\text { CROATIA- } \\
\text { Korcula }\end{array}$ & Caucasian & $\begin{array}{l}909 \\
926\end{array}$ & 0.52 & 0.15 & 0.07 & $5.0 \times 10^{-2}$ & 0.029 & 0.021 & 0.17 & -0.13 & 0.12 & 0.28 \\
\hline & & $\begin{array}{l}\text { CROATIA- } \\
\text { Split }\end{array}$ & Caucasian & $\begin{array}{l}445 \\
422\end{array}$ & 0.59 & 0.01 & 0.06 & 0.94 & 0.023 & 0.019 & 0.22 & 0.09 & 0.12 & 0.43 \\
\hline & & RAINE & Caucasian & 108 & 0.56 & -0.02 & 0.12 & 0.85 & -0.032 & 0.228 & 0.89 & 0.07 & 0.25 & 0.78 \\
\hline & & $\begin{array}{l}\text { Meta } \\
\text { Caucasian }\end{array}$ & - & $\begin{array}{l}2,690 \\
2,007\end{array}$ & - & 0.10 & 0.03 & $2.0 \times 10^{-3}$ & 0.028 & 0.014 & $4.1 \times 10^{-2}$ & 0.05 & 0.05 & 0.26 \\
\hline & & Meta ALL ${ }^{\S}$ & - & $\begin{array}{l}11,064 \\
10,988\end{array}$ & - & 0.12 & 0.02 & $3.9 \times 10^{-13}$ & 0.051 & 0.004 & $2.7 \times 10^{-40}$ & 0.04 & 0.03 & 0.24 \\
\hline
\end{tabular}

$E A F$, effect allele frequency; $S E$, standard error.

$P$ values derived with linear regression and inverse variance meta-analysis.

*Each abbreviation is clarified in Supplementary Methods.

$\dagger$ Axial length was not available in SP2.

$\ddagger$ Corneal curvature was not available in ORCADES.

\$Meta-analysis of Asians (discovery and replication) and Caucasian.

Asian cohorts

(stratified by WNT7B rs10453441)

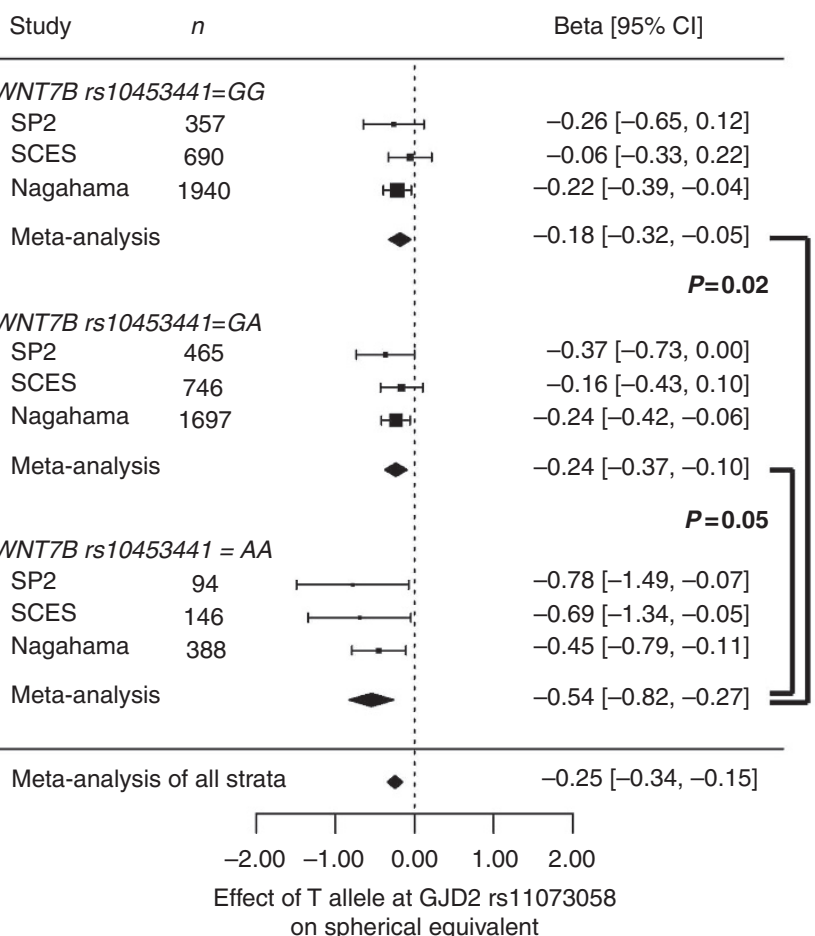

Caucasian cohorts

(stratified by WNT7B rs10453441)

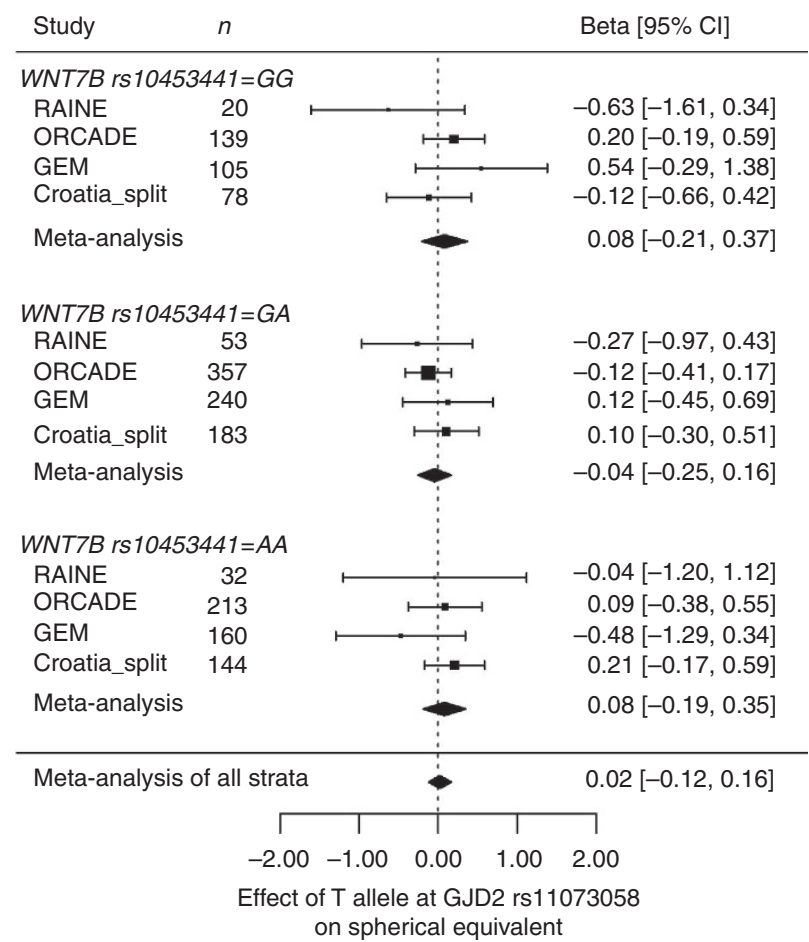

Figure 2 | Assessment of interactive effect between WNT7B and GJD2 and association with the spherical equivalent. In Asian cohorts, the effects of GJD2 rs11073058 on the spherical equivalent were highest when the individual had the homozygous variant (AA genotype) at WNT7B rs10453441 $(\beta=-0.54 \mathrm{D} ; 95 \% \mathrm{Cl}=-0.82$ to $-0.27 \mathrm{D})$, compared with individuals with the AG genotype $(\beta=-0.24 \mathrm{D} ; 95 \% \mathrm{Cl}=-0.37$ to $-0.10 \mathrm{D} ; \mathrm{P}=0.05$ by $z$-test) or those with the $\mathrm{GG}$ genotype $(\beta=-0.18 \mathrm{D} ; 95 \% \mathrm{Cl}=-0.32$ to $-0.05 \mathrm{D} ; P=0.02$ by $z$-test). This trend was not observed in Caucasian cohorts. 
95\% CI: -0.37 to $-0.10 \mathrm{D})$ or in those with the GG genotype $(\beta=-0.18 \mathrm{D} ; 95 \% \mathrm{CI}$ : -0.32 to $-0.05 \mathrm{D})$. The genotypic model revealed marginal effect-measure modification $\left(P_{\text {genotypic }(\text { analysis of variance })}=0.07\right)$, with post hoc $P$ values of 0.02 (AA versus GG), 0.05 (AA versus AG) and 0.60 (AG versus GG) based on $z$-test. On the other hand, the recessive model of effect-measure modification was statistically significant $\left(P_{\text {recessive }}=0.024\right)$ as the effect of GJD2 rs11073058 $\mathrm{T}$ allele on spherical equivalent was $-0.21 \mathrm{D}(95 \% \mathrm{CI}:-0.11$ to $-0.31 \mathrm{D})$ in individuals with non-AA genotype at WNT7B rs10453441.

Clinical relevance of $W N T 7 B$ to extreme myopia. To evaluate the possible association between $W N T 7 B$ and extreme myopia, we performed a case-control study. We found significant association between WNT7B rs10453441 and extreme myopia (patients with average axial length of $\geq 28 \mathrm{~mm}$ ) in the Japanese cohort (odds ratio $=1.14 \quad(95 \% \quad \mathrm{CI}=1.02-1.27), \quad P_{\text {meta- }}$ Japanese $=0.018)$. Although the association in Chinese cohorts was not statistically significant $\left(P_{\text {meta-Chinese }}=0.30\right)$, the effect size and its direction were similar to those in Japanese cohort (odds ratio $=1.10(95 \% \mathrm{CI}=0.92-1.30))$. The lack of association might be due to the limited sample size of extreme myopia individuals (1,064 Japanese and 414 Chinese) as these results were consistent $\left(I^{2}=0 \%, \quad P_{\text {heterogeneity }}=0.84\right)$. Indeed, overall meta-analysis revealed more significant association (odds ratio $=1.13(95 \%$ $\left.\mathrm{CI}=1.03-1.24), P_{\text {meta-all }}=0.011\right)$. These results are summarized in Table 4 and Supplementary Fig. 2.

The expression of WNT7B in experimental myopia mice. Our immunohistochemical study of the mouse cornea showed that WNT7B was predominantly expressed in the endothelial cell layer (Fig. 3a). In contrast to corneal studies, the role of WNT signaling in the posterior pole has been examined in various parts of the tissue, such as the neural retina, retinal vasculature and retinal pigment epithelium ${ }^{24-28}$. To evaluate the expression pattern of WNT7B in the posterior pole of eyes, we examined the localization of WNT7B in the mouse eye. In the retina of a 21-day-old male mouse, WNT7B expression was observed predominantly in ganglion cells (Fig. 3b). Ganglion cells were also stained with the ganglion cell marker Brn-3a. Not all ganglion cells expressed Brn3a, and hence we could not confirm expression of WNT7B in all ganglion cells, but we could confirm that all ganglion cells expressing Brn-3a also co-expressed WNT7B.

To explore the roles of WNT7B in myopia development, we evaluated the expression of the $W N T 7 B$ gene in the cornea and the neural retina of myopia-induced mice (Fig. 4). WNT7B mRNA levels were significantly higher in the retinas of myopic eyes than those of corresponding control eyes $(P=0.016)$. In
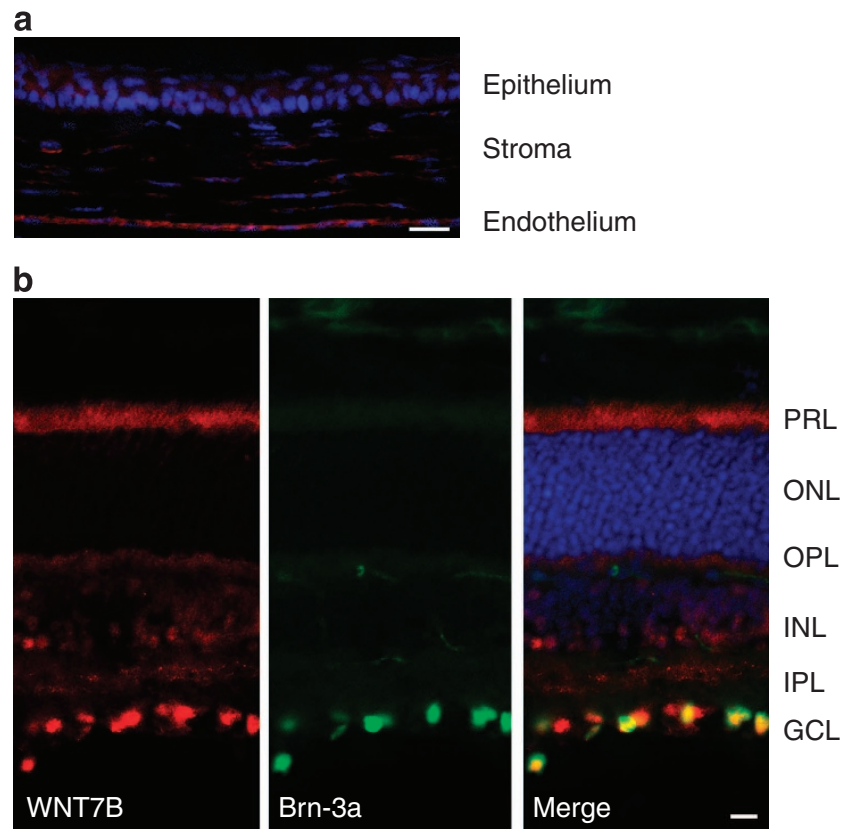

Figure 3 | Expression of WNT7B in retinal ganglion cells of C57BL/6 mice. (a) Mouse corneal sections were immunostained with antibodies against WNT7B (red). The upper part of the panel shows the corneal epithelium. (b) Mouse retinal sections were immunostained with antibodies against WNT7B (red) and Brn-3a (green). The nuclei are counterstained with 4',6-diamidino-2-phenylindole (blue) in the merged image. The upper part of the panel shows the retinal pigment epithelium side, while the lower panel shows the vitreous side. WNT7B expression was observed in ganglion cells stained with Brn-3a. Scale bar, $10 \mu \mathrm{m}$. GCL, ganglion cell layer; INL, inner nuclear layer; IPL, inner plexiform layer; ONL, outer nuclear layer; $\mathrm{OPL}$, outer plexiform layer; PRL, photoreceptor layer.

Table 4 | Association of WNT7B rs10453441 with extreme myopia.

\begin{tabular}{|c|c|c|c|c|c|c|c|c|c|c|c|c|c|c|c|}
\hline \multirow[t]{3}{*}{ Ethnicity } & \multirow[t]{3}{*}{ Collection } & \multicolumn{6}{|c|}{ Control $^{\star}$} & \multicolumn{6}{|c|}{ Extreme myopia ${ }^{\dagger}$} & \multirow{3}{*}{$\begin{array}{l}\text { Odds ratio } \\
(95 \% \mathrm{Cl})^{\ddagger}\end{array}$} & \multirow{3}{*}{$\begin{array}{c}P \\
\text { value }\end{array}$} \\
\hline & & \multirow[t]{2}{*}{$n$} & \multirow{2}{*}{$\begin{array}{c}\text { SE [D] }]^{\S} \text { median (first, } \\
\text { third quartile) }\end{array}$} & \multicolumn{3}{|c|}{ rs10453441 } & \multirow{2}{*}{$\begin{array}{c}\text { A } \\
\text { allele } \\
\text { freq. }\end{array}$} & \multirow[t]{2}{*}{$n$} & \multirow{2}{*}{$\begin{array}{c}\text { SE [D] }{ }^{\S} \text { median } \\
\text { (first, third quartile) }\end{array}$} & \multicolumn{3}{|c|}{ rs10453441 } & \multirow{2}{*}{$\begin{array}{c}\text { A } \\
\text { allele } \\
\text { freq. }\end{array}$} & & \\
\hline & & & & GG & GA & AA & & & & GG & GA & AA & & & \\
\hline \multirow[t]{2}{*}{ Japanese } & $\begin{array}{l}\text { Nagahama/ } \\
\text { Kyotoll }\end{array}$ & 1,902 & $0.03(-0.69,0.81)$ & 952 & 781 & 169 & 0.29 & 938 & $-13.50(-16.50,-11.25)$ & 449 & 376 & 113 & 0.32 & $1.13(1.00-1.28)$ & 0.043 \\
\hline & $\begin{array}{l}\text { Yokohama } \\
\text { Meta }\end{array}$ & $\begin{array}{c}395 \\
2,297\end{array}$ & $\begin{array}{c}0.375(0.125,0.875) \\
-\end{array}$ & $\begin{array}{l}173 \\
-\end{array}$ & $\begin{array}{l}178 \\
-\end{array}$ & $\begin{array}{l}44 \\
-\end{array}$ & $\begin{array}{c}0.34 \\
-\end{array}$ & $\begin{array}{c}109 \\
1,064\end{array}$ & $\begin{array}{c}-11.62(-13.38,-10.00) \\
-\end{array}$ & $\begin{array}{l}43 \\
-\end{array}$ & $\begin{array}{l}48 \\
-\end{array}$ & $\frac{18}{-}$ & $\begin{array}{c}0.38 \\
-\end{array}$ & $\begin{array}{l}1.23(0.90-1.68) \\
1.14(1.02-1.27)\end{array}$ & $\begin{array}{l}0.18 \\
0.018\end{array}$ \\
\hline Chinese & $\begin{array}{l}\text { SCES } \\
\text { Hong Kong } \\
\text { Sichuan } \\
\text { Meta }\end{array}$ & $\begin{array}{c}1,493 \\
422 \\
477 \\
2,392\end{array}$ & $\begin{array}{c}0.44(-0.31,1.31) \\
0.19(-0.13,0.50) \\
0.0(-0.63,0.50) \\
-\end{array}$ & $\begin{array}{c}668 \\
186 \\
224 \\
-\end{array}$ & $\begin{array}{r}679 \\
186 \\
211 \\
-\end{array}$ & $\begin{array}{r}146 \\
50 \\
42 \\
-\end{array}$ & $\begin{array}{l}0.32 \\
0.33 \\
0.30 \\
-\end{array}$ & $\begin{array}{r}22 \\
257 \\
135 \\
414\end{array}$ & $\begin{array}{c}-11.73(-13.56,-8.06) \\
-11.19(-12.95,-9.86) \\
-13.00(-17.00,-10.00) \\
-\end{array}$ & $\begin{array}{r}6 \\
114 \\
57 \\
-\end{array}$ & $\begin{array}{r}14 \\
106 \\
67 \\
-\end{array}$ & $\begin{array}{r}2 \\
37 \\
11 \\
-\end{array}$ & $\begin{array}{l}0.40 \\
0.35 \\
0.32 \\
-\end{array}$ & $\begin{array}{l}1.44(0.74-2.74) \\
1.05(0.71-1.55) \\
1.10(0.68-1.68) \\
1.10(0.92-1.30)\end{array}$ & $\begin{array}{l}0.20 \\
0.68 \\
0.51 \\
0.30\end{array}$ \\
\hline Meta & $\begin{array}{l}5 \\
\text { Collections }\end{array}$ & 4,689 & - & - & - & - & - & 1,478 & - & - & - & - & - & $1.13(1.03-1.24)$ & 0.011 \\
\hline \multicolumn{16}{|c|}{$\begin{array}{l}\mathrm{Cl} \text {, confidence interval; } \mathrm{D} \text {, diopter; } \mathrm{SE} \text {, spherical equivalent. } \\
P \text { values derived using chi-squared test for tend and inverse variance meta-analysis. } \\
{ }^{*} \text { Controls: } 22 \mathrm{~mm} \leq \text { axial length } \leq 24 \mathrm{~mm} \text { except for Sichuan; }-1 \mathrm{D} \leq \text { spherical equivalent } \leq 1 \mathrm{D} \text { for Sichuan. } \\
\dagger \text { Extreme myopia: axial length } \geq 28 \mathrm{~mm} \text {. } \\
\ddagger \text { Based on the logistic regression analysis. } \\
\text { §Only phakic samples whose refraction were available. } \\
\| \text { Controls from Nagahama study and cases from Kyoto high myopia cohort. }\end{array}$} \\
\hline
\end{tabular}



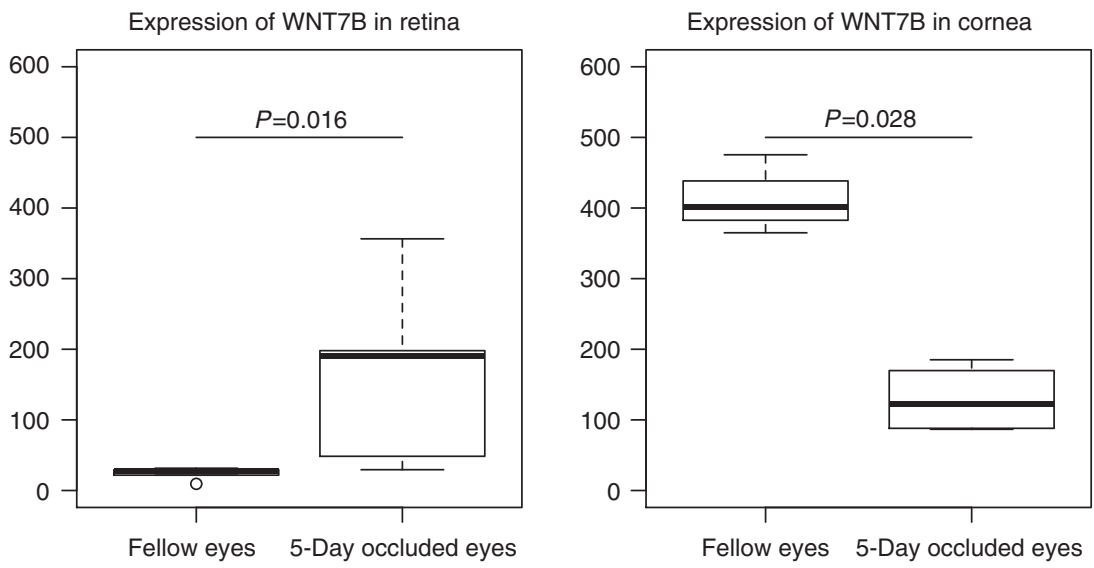

Figure 4 | Differential expression of WNT7B between treated eyes and fellow eyes in experimental myopia mice $(\boldsymbol{n}=\mathbf{5}$ in each arm). The vertical axis indicates WNT7B expression normalized to $\beta$-actin expression. WNT7B expression in the retina was significantly upregulated in the experimental myopia eyes $(P=0.016$ by Wilcoxon test), but was significantly downregulated in the cornea $(P=0.028$ by Wilcoxon test).

contrast, $W N T 7 B$ mRNA levels were significantly reduced in the corneas of myopic eyes $(P=0.029)$.

\section{Discussion}

We performed a GWAS in a large Japanese cohort and found that $W N T 7 B$ was significantly associated with axial length and corneal curvature. The associations with axial length and corneal curvature were successfully replicated in both Chinese and Caucasian cohorts. In addition, the WNT7B genotype strengthened the effects of GJD2, a widely replicated myopia susceptibility gene ${ }^{11,23}$. Furthermore, the WNT7B polymorphism rs10453441 was also significantly associated with extreme myopia. Our findings that WNT7B expression was downregulated in the cornea and upregulated in retinal ganglion cells in experimental myopia suggested that WNT7B plays a key role in controlling myopia development through compensatory mechanisms.

Although many candidate gene studies ${ }^{29-33}$ and GWASs $^{10-12,34-39}$ have been conducted to investigate myopiaassociated traits, little is known about the pivotal pathways involved in the development of myopia. The largest genome-wide meta-analysis conducted by CREAM ${ }^{11}$ on refractive error and a recent large GWAS conducted by $23 \mathrm{andMe}{ }^{39}$ on age of myopia onset reported a total of 42 loci associated with myopia. Many of them were associated with signaling cascades in the neural retina, retinal pigment epithelium, choroid and sclera. However, not all the susceptibility genes were detected by previous GWASs because it is difficult to detect SNPs with low minor-allele frequencies or small effect sizes and because SNPs that were not included in the commercially available assay could not be evaluated. Even though a genomic imputation technique was used, the imputation quality was not necessarily high as it is dependent on whether imputed SNPs are in high LD with directly genotyped SNPs. To overcome this problem, we applied the dense $2.5 \mathrm{M}$ chip for $53.7 \%$ of the samples and a $610 \mathrm{~K}$ chip for $49.3 \%$ of the samples, which were imputed to the intra-cohort reference panel (296 samples from the Nagahama Study cohort genotyped using all platforms). This strategy provided a dense, high-quality data set and enabled us to find a novel susceptibility gene for corneal curvature and axial length. The novel susceptibility gene, $W N T 7 B$, had stronger effects on axial length $(\beta=0.13)$ than the established susceptibility gene, GJD2 ( $\beta=0.09$ in the current study and $\beta=0.06-0.07$ in a previous report ${ }^{12}$ ).

Although the genotypes of the SNPs within WNT7B were not directly associated with refractive error in the Nagahama Study, which only included a small number of subjects with extreme myopia (49 individuals in first and second stage), SNPs within $W N T 7 B$ displayed significant associations with extreme myopia in our case-control studies. These data suggest that the effects of WNT7B might become apparent when the compensatory mechanisms of eye components to control refractive error are disrupted, resulting in extreme myopia. Therefore, we investigated the interactive effect of $W N T 7 B$ on GJD2 for refractive error. The effects of the T-risk allele of rs11073058 in GJD2 on myopia were the largest when the genotype of WNT7B rs10453441 was AA. As the reported effect size of GJD2 on refraction ranged from $-0.10 \mathrm{D}$ to $-0.12 \mathrm{D}$ (refs 12,40), the acceleration of myopia by $W N T 7 B$ up to $-0.54 \mathrm{D}$ per copy of the $\mathrm{T}$ allele at GJD2 rs11073058 in Asians is of note, which is statistically significant in the recessive model. Interestingly, this interactive effect was not apparent in Caucasian samples. This might be due to the limited sample size of the Caucasian cohorts, allelic differences between Asians and Caucasians, or it might, in part, explain the higher prevalence of myopia in Asians.

In a mouse model, WNT7B was downregulated in the cornea and upregulated in the retina of experimental myopic eyes. Considering that the effect of WNT7B rs10453441 on myopia through axial length is in the opposite direction to that through corneal curvature, WNT7B may be under strict control to correlate the anterior and posterior segments in the emmetropization of the eye. To date, accumulating evidence suggests that retinal image defocus is the trigger for myopia, and the role of amacrine cells in delivering an image defocus signal has been intensively investigated in the context of myopia development $^{20,21,41}$. Nevertheless, the precise mechanisms mediating the transduction of this defocus signal leading to myopia are still unclear. GJD2 is reportedly localized at the synapses between alpha-ganglion cells, AII amacrine cells, cone bipolar cells and photoreceptor cells ${ }^{42-51}$. Of all the interactions between these cells, the coupling between the amacrine cells and ganglion cells has been reported to be dramatically reduced in GJD2 knockout mice ${ }^{52}$. Together with our findings indicating that WNT7B was localized to retinal ganglion cells and that its expression was significantly upregulated in experimental myopic eyes, these data support the hypothesis that WNT7B and GJD2 co-operatively regulate the normal growth of the eye through interactions between the alpha-ganglion cells and AII amacrine cells. Interestingly, alpha-ganglion cells are predominantly observed in the peripheral retina and have large dendritic regions to receive inputs from amacrine cells ${ }^{47,53-57}$. The hypothesis that alpha- 
ganglion cells and AII amacrine cells are implicated in emmetropization is consistent with the peripheral defocus theory, a theory of myopia development that has been supported by clinical evidence ${ }^{58-61}$.

Although the current study revealed for the first time the possible involvement of $W N T 7 B$ in myopia development, the involvement of Wnt signaling itself in this process has been suggested previously. For example, we showed that polymorphisms in PAX6 and $\delta$-catenin (CTNND2) are associated with high-grade myopia ${ }^{29,32,33,35}$. PAX6 can directly control both CTNND2 and WNT7B expression ${ }^{62,63}$, and both molecular pathways can result in increased $\beta$-catenin nuclear translocation to activate Wnt signaling ${ }^{64,65}$. Independently, the CREAM consortium found that polymorphisms in RSPO1 and ZNRF3, which regulate Wnt signaling by controlling the turnover of Wnt receptors, were significantly associated with axial length ${ }^{12,66}$. The present study suggests that WNT7B can be involved in the development of myopia. Further studies are needed to investigate interactions between genes in the same or different cascades of Wntsignaling pathways. Better understanding of the pathophysiology underlying myopia will guide interventions for prevention and treatment.

In summary, we have demonstrated significant association of $W N T 7 B$ with corneal curvature and axial length in three different ethnic groups and found that $W N T 7 B$ could facilitate the development of myopia through cooperation with GJD2. Furthermore, the $W N T 7 B$ polymorphism rs10453441 was significantly associated with extreme myopia in patients with Asian ancestry. In the retina, WNT7B was localized to ganglion cells, and its expression was significantly increased in mice with experimental myopia, whereas in the cornea its expression was localized to the endothelium and its expression downregulated in experimental myopia. We, therefore, propose that Wnt-signaling pathways that are vital for coordinating vision and disruption in these pathways leads to myopia.

\section{Methods}

Patient enrollment. There were 9,804 Japanese participants in the Nagahama Prospective Genome Cohort for Comprehensive Human Bioscience (the Nagahama Study). The Nagahama Study cohort was recruited from 2008 to 2010 from the general population living in Nagahama City, Shiga Prefecture, Japan ${ }^{67,68}$. All ophthalmological measurements and blood sampling were performed at the time of enrollment. Genomic DNA was extracted from peripheral blood samples by the phenol-chloroform method. All study procedures were approved by the Ethics Committee of Kyoto University Graduate School of Medicine.

For the replication stage, we also included seven other cohorts that consisted of five Caucasian cohorts and two Singaporean Chinese cohorts. Detailed information and sample collections for these cohorts can be found in the Supplementary Methods. All patients were enrolled in the study after giving informed consent, ethical approval was granted by the SingHealth Centralized Institutional Review Board (cohorts SP2 and SCES), the Ethics Committee of the Medical School, University of Split and the NHS Lothian South East Scotland Research Ethics Committee (CROATIA-Split and CROATIA-Korcula) and the NHS Orkney Research Ethics Committee and the North of Scotland Research Ethics Committee (ORCADES). The Human Research and Ethics Committee of the Royal Victorian Eye and Ear Hospital, Melbourne (GEM) and the King Edward Memorial Hospital and Princess Margaret Hospital for Children ethics boards (RAINE). Genomic DNA was extracted from peripheral blood samples according to standard laboratory procedures.

Individuals who had undergone ocular surgery (with the exception of cataract surgery) and who had undergone ocular laser treatment were excluded from the analysis of all three traits. In addition, individuals who had undergone cataract surgery were excluded from analyses of the spherical equivalent and corneal curvature.

Evaluation of ophthalmological measurements. All the participants in the Nagahama Study had their axial length (millimeter (mm); IOL Master, Carl Zeiss Meditec, Dublin, CA, USA), spherical equivalent (diopter (D); ARK-530A, Nidek, Aichi, Japan) and corneal curvature (mm; ARK-530A, Nidek) measured for both eyes. Colour fundus photographs were also obtained from all the participants (CRDG10, Canon, Tokyo, Japan). History of cataract surgery, ocular surgery other than cataract surgery and ocular laser treatment, including photocoagulation, was obtained using a questionnaire. For the replication cohorts, detailed information is given in the Supplementary Methods.
Genome-wide SNP genotyping. Genome-wide SNP genotyping was performed on samples from 3,710 participants who joined the Nagahama cohort from 2008 to 2009 A series of BeadChip DNA arrays, namely HumanHap610 Quad (1,828 samples), HumanOmni2.5-4 (1,616 samples), HumanOmni2.5-8 (378 samples), HumanOmni2.5s (192 samples) and HumanExome (192 samples; Illumina, San Diego, CA, USA) were used for the analysis. Some of the samples were repeatedly genotyped using different arrays, and 296 samples that had been genotyped using HumanHap610 Quad, HumanOmni2.5-8, HumanOmni2.5s and HumanExome were used as a reference panel in the genotype imputation. SNPs with a call rate $<99 \%$, MAF $<1 \%$ and significant deviation from Hardy-Weinberg Equilibrium ( $P$ value for deviation $<1.0 \times 10^{-7}$ ) were excluded from further statistical analysis.

Samples with a call rate $<95 \%(n=162)$ were excluded from the analysis. Among the remaining 3,548 subjects, 295 estimated to have a first- or seconddegree kinship within this population (pi-hat $>0.35$, PLINK ver. 1.07 (http:// pngu.mgh.harvard.edu/ purcell/plink/)) and seven ancestry outliers identified by principal component analysis with the HapMap Phase 2 release 28 JPT data set as a reference (EIGENSTRAT ver. 2.0 (http://www.hsph.harvard.edu/alkes-price/ software/)) were also excluded from the analysis.

Genotype imputation was performed using MACH ver. 1.0.16 software (http:// www.sph.umich.edu/csg/abecasis/MACH/tour/imputation.html) and 1,792,015 SNPs from the 296-sample reference panel. Imputed SNPs for which the MAF was $<0.01 \%$ or $R^{2}<0.5$ were excluded from the following association analysis. Finally, $1,773,334$ SNPs from 3,248 individuals were fixed.

Replication genotyping. We conducted a two-stage replication study. The first stage was the replication within the Nagahama Study participants and the second stage was the trans-ethnic replication.

For the first stage, we genotyped the subset of remaining samples from the Nagahama Study $(n=3,460)$ whose phenotypes were all available using TaqMan allelic discrimination probes (Applied Biosystems). For the second stage, genotypes of rs200329677 and/or rs10453441 were determined by one of the following methods: using the HumanOmni1M chip (Illumina), using TaqMan allelic discrimination probes (Applied Biosystems) or imputation from the existing data The particular method used is described in Supplementary Table 2. We first used direct genotyping data. However, if only imputed data were available, we included them only when the imputation quality $\left(R^{2}\right)$ was $>0.5$.

Statistical analysis. Genome-wide QTL analysis was conducted for the three myopia-related traits: axial length, spherical equivalent and corneal curvature. We used the mean values of both eyes for each trait. If one eye was not applicable, measurement of the other eye was used in the analysis. For every post-QC SNP, we evaluated the association between the genotypes and the trait using a multivariable linear regression, assuming an additive model. This regression framework allowed us to adjust for covariates such as age, sex and height. Experimental-wide significance was set at $2.82 \times 10^{-8}$, corresponding to $P=0.05$ divided by the $1,773,334$ SNPs included in the QTL analysis. We also carried SNPs with $P$ values $<1.00 \times 10^{-6}$ forward to the replication stage if their MAFs were at least $5 \%$. Meta-analysis was conducted using inverse variance weights for 8,981 Asians, 2,835 Caucasians and all subjects. This process was performed using the METAL software (http://www.sph.umich.edu/csg/abecasis/Metal/). Estimates of effect size with standard error were compared using the $z$-test. Manhattan plots and forest plots (package 'metafor') were generated using R version 2.15 (http://www.r-project.org)

Association with extreme myopia. To investigate the association between $W N T 7 B$ rs10453441 and extreme myopia, we performed two association tests. In these analyses, we defined extremely myopic patients as patients whose average axial lengths in both eyes were $>28.00 \mathrm{~mm}$, according to a previous report ${ }^{9}$. Samples whose average axial lengths ranged from 22.00 to $24.00 \mathrm{~mm}$ were used as control samples.

We used two case-control data sets of the Japanese population. The first one was the Kyoto/Nagahama data set consisting of 938 highly myopic cases recruited from the Kyoto High Myopia cohort $9,29,30$ and 1,902 controls extracted from the Nagahama Study samples genotyped by the TaqMan method. The Kyoto High Myopia samples were also genotyped using the TaqMan method. The second cohort consisted of Yokohama samples, recruited from Yokohama City University, which contained 109 extreme myopia patients and 395 controls. They were both genotyped using Illumina OmniExpress at Yokohama City University. The demographics of the Kyoto High Myopia cohort and the Yokohama samples are shown in Supplementary Table 3. For further replication, three Chinese cohorts, that is, SCES, Hong Kong and Sichuan, were used for the same analysis. Chinese samples were also genotyped using the TaqMan method.

The analyses were performed using logistic regression. Meta-analyses were conducted using inverse-variance weights for two Japanese cohorts, three Chinese cohorts and all five cohorts.

Experimental myopia mouse model. To induce form-deprivation myopia in mice ${ }^{16,69,70}$, we occluded the right eyes of male C57BL/6 mice (Japan SLC Inc., Shizuoka, Japan) with diffuser goggles from postnatal day 21 . The mice were housed individually in standard mouse cages for 5 days at $25^{\circ} \mathrm{C}$ on a $12 \mathrm{~h}: 12 \mathrm{~h}$ light:dark cycle, with standard rodent chow pellets and water freely available. The 
animal study was approved by the Kyoto University Animal Experimentation Committee. All the procedures performed in this study complied with the Association of Research in Vision and Ophthalmology Statement for the Use of Animals in Ophthalmology and Vision Research.

Immunohistochemical staining. After a mouse was killed, eyes were enucleated and fixed with $4 \%$ paraformaldehyde in $0.1 \mathrm{M}$ PB for $20 \mathrm{~min}$. The expression of WNT7B in the retina was examined in vertical sections. For this, the eyes were cryoprotected in a sucrose gradient ( 10 and $30 \% \mathrm{w} / \mathrm{v}$ sucrose in $0.1 \mathrm{M} \mathrm{PB})$, and $14-\mu \mathrm{m}$ cryostat sections were cut. The sections were blocked for $1 \mathrm{~h}$ in a solution containing 10\% normal goat serum (Millipore), $0.3 \%$ Triton X-100 (Bio-Rad, Hercules, CA, USA) and 0.1 M PB. The primary antibodies were diluted in the blocking solution and incubated overnight at $4{ }^{\circ} \mathrm{C}$, followed by incubation for $1 \mathrm{~h}$ in the secondary antibody solution. The primary antibodies used were 1:200 dilution of rabbit anti-Wnt7b (NOVUS, NBP1-59564) and 1:100 dilution of mouse antiBrn-3a (CHEMICON, MAB1585). For secondary antibodies, 1:1,000 dilutions of Alexa Fluor 594 goat anti-rabbit IgG $(\mathrm{H}+\mathrm{L})$ and Alexa Fluor 488 goat anti-mouse IgG $(\mathrm{H}+\mathrm{L})$ antibodies (catalogue numbers A-11012 and A-11001, respectively, Invitrogen, Eugene, OR, USA) were used. All the steps were carried out at $4{ }^{\circ} \mathrm{C}$. All the images were obtained using a KEYENCE BZ-9000 microscope.

Quantitative real-time polymerase chain reaction. To evaluate changes in the expression level of WNT7B at the early stage of myopia, we used experimental mice treated for 5 days. Gene expression in the corneas and retinas of treated and untreated eyes were compared using a Wilcoxon test.

Total RNA was isolated from mouse corneas or neural retinas using QIAzol Reagent (Qiagen, Tokyo, Japan) for the experimental eyes $(n=5)$ and untreated eyes $(n=5)$. RNA was purified using the RNeasy Micro kit (Qiagen).

Purified RNA was reverse transcribed into cDNA by using a ReverTra Ace quantitative real-time polymerase chain reaction (qPCR) RT Kit (Toyobo, Hiroshima, Japan). We performed qPCR using ThunderBird SYBR qPCR mix (Toyobo) to validate the gene expression. The sequences of the QPCR primers are shown in Supplementary Table 4. The reactions were run using an ABI Prism 7300 Sequence Detection System (Applied Biosystems, Foster City, CA, USA) for 40 cycles under the following conditions: $95^{\circ} \mathrm{C}$ for $15 \mathrm{~s}$ and $60^{\circ} \mathrm{C}$ for $60 \mathrm{~s}$. To normalize for input load of cDNA between samples, $\beta$-actin was used as an endogenous internal control. Threshold cycle (CT) was determined automatically, and relative change in mRNA expression was calculated using $\Delta \Delta \mathrm{CT}$ values. All cDNA samples were tested in triplicate and the average values were used in statistical analysis.

\section{References}

1. Saw, S. M., Gazzard, G., Shih-Yen, E. C. \& Chua, W. H. Myopia and associated pathological complications. Ophthalmic Physiol. Opt. 25, 381-391 (2005).

2. Wong, T. Y., Foster, P. J., Johnson, G. J. \& Seah, S. K. Education, socioeconomic status, and ocular dimensions in Chinese adults: the Tanjong Pagar Survey. $\mathrm{Br}$. J. Ophthalmol. 86, 963-968 (2002).

3. Sawada, A., Tomidokoro, A., Araie, M., Iwase, A. \& Yamamoto, T. Refractive errors in an elderly Japanese population: the Tajimi study. Ophthalmology 115, 363-370 e3 (2008).

4. Vitale, S., Sperduto, R. D. \& Ferris, 3rd F. L. Increased prevalence of myopia in the United States between 1971-1972 and 1999-2004. Arch. Ophthalmol. 127, 1632-1639 (2009).

5. Resnikoff, S., Pascolini, D., Mariotti, S. P. \& Pokharel, G. P. Global magnitude of visual impairment caused by uncorrected refractive errors in 2004. Bull. World Health Organ. 86, 63-70 (2008).

6. Gonzalez Blanco, F., Sanz Fernandez, J. C. \& Munoz Sanz, M. A. Axial length, corneal radius, and age of myopia onset. Optom. Vis. Sci. 85, 89-96 (2008).

7. Guggenheim, J. A. et al. A genome-wide association study for corneal curvature identifies the platelet-derived growth factor receptor alpha gene as a quantitative trait locus for eye size in white Europeans. Mol. Vis. 19, 243-253 (2013).

8. Miyake, M. et al. Vascular endothelial growth factor gene and the response to anti-vascular endothelial growth factor treatment for choroidal neovascularization in high myopia. Ophthalmology 121, 225-233 (2014).

9. Miyake, M. et al. Evaluation of pigment epithelium-derived factor and complement factor I polymorphisms as a cause of choroidal neovascularization in highly myopic eyes. Invest. Ophthalmol. Vis. Sci. 54, 4208-4212 (2013).

10. Khor, C. C. et al. Genome-wide association study identifies ZFHX1B as a susceptibility locus for severe myopia. Hum. Mol. Genet. 22, 5288-5294 (2013).

11. Verhoeven, V. J. et al. Genome-wide meta-analyses of multiancestry cohorts identify multiple new susceptibility loci for refractive error and myopia. Nat. Genet. 45, 314-318 (2013).

12. Cheng, C. Y. et al. Nine loci for ocular axial length identified through genomewide association studies, including shared loci with refractive error. Am. J. Hum. Genet. 93, 264-277 (2013).

13. Han, S. et al. Association of variants in FRAP1 and PDGFRA with corneal curvature in Asian populations from Singapore. Hum. Mol. Genet. 20, 3693-3698 (2011)
14. Norton, T. T. \& Siegwart, Jr. J. T. Light levels, refractive development, and myopia--a speculative review. Exp. Eye Res. 114, 48-57 (2013).

15. Irving, E. L., Callender, M. G. \& Sivak, J. G. Inducing myopia, hyperopia, and astigmatism in chicks. Optom. Vis. Sci. 68, 364-368 (1991).

16. Barathi, V. A., Boopathi, V. G., Yap, E. P. \& Beuerman, R. W. Two models of experimental myopia in the mouse. Vision Res. 48, 904-916 (2008).

17. Smith, 3rd E. L., Hung, L. F. \& Huang, J. Relative peripheral hyperopic defocus alters central refractive development in infant monkeys. Vision Res. 49, 2386-2392 (2009).

18. Smith, 3rd E. L., Kee, C. S., Ramamirtham, R., Qiao-Grider, Y. \& Hung, L. F. Peripheral vision can influence eye growth and refractive development in infant monkeys. Invest. Ophthalmol. Vis. Sci. 46, 3965-3972 (2005).

19. Smith, 3rd E. L. et al. Effects of foveal ablation on emmetropization and formdeprivation myopia. Invest. Ophthalmol. Vis. Sci. 48, 3914-3922 (2007).

20. Fischer, A. J., McGuire, J. J., Schaeffel, F. \& Stell, W. K. Light- and focusdependent expression of the transcription factor ZENK in the chick retina. Nat. Neurosci. 2, 706-712 (1999).

21. Schippert, R., Burkhardt, E., Feldkaemper, M. \& Schaeffel, F. Relative axial myopia in Egr-1 (ZENK) knockout mice. Invest. Ophthalmol. Vis. Sci. 48, 11-17 (2007).

22. Baird, P. N., Schache, M. \& Dirani, M. The GEnes in Myopia (GEM) study in understanding the aetiology of refractive errors. Prog. Retin. Eye Res. 29, 520-542 (2010).

23. Solouki, A. M. et al. A genome-wide association study identifies a susceptibility locus for refractive errors and myopia at 15q14. Nat. Genet. 42, 897-901 (2010).

24. Sanges, D. et al. Wnt/beta-catenin signaling triggers neuron reprogramming and regeneration in the mouse retina. Cell Rep. 4, 271-286 (2013).

25. Liu, B. et al. Wnt signaling promotes Muller cell proliferation and survival after injury. Invest. Ophthalmol. Vis. Sci. 54, 444-453 (2013).

26. Bharti, K. et al. A regulatory loop involving PAX6, MITF, and WNT signaling controls retinal pigment epithelium development. PLoS Genet. 8, e1002757 (2012).

27. Del Debbio, C. B. et al. Notch and Wnt signaling mediated rod photoreceptor regeneration by Muller cells in adult mammalian retina. PLOS ONE 5, e12425 (2010).

28. Liu, H., Thurig, S., Mohamed, O., Dufort, D. \& Wallace, V. A. Mapping canonical Wnt signaling in the developing and adult retina. Invest. Ophthalmol. Vis. Sci. 47, 5088-5097 (2006).

29. Miyake, M. et al. Association of paired box 6 with high myopia in Japanese. Mol. Vis. 18, 2726-2735 (2012).

30. Miyake, M. et al. Insulin-like growth factor 1 is not associated with high myopia in a large Japanese cohort. Mol. Vis. 19, 1074-1081 (2013).

31. Khor, C. C. et al. Support for TGFB1 as a susceptibility gene for high myopia in individuals of Chinese descent. Arch. Ophthalmol. 128, 1081-1084 (2010).

32. Han, W. et al. Association of PAX6 polymorphisms with high myopia in Han Chinese nuclear families. Invest. Ophthalmol. Vis. Sci. 50, 47-56 (2009).

33. Jiang, B. et al. PAX6 haplotypes are associated with high myopia in Han chinese. PLoS ONE 6, el9587 (2011).

34. Nakanishi, H. et al. A genome-wide association analysis identified a novel susceptible locus for pathological myopia at 11q24.1. PLoS Genet. 5, e1000660 (2009).

35. Li, Y. J. et al. Genome-wide association studies reveal genetic variants in CTNND2 for high myopia in Singapore Chinese. Ophthalmology 118, 368-375 (2011).

36. Shi, Y. et al. Genetic variants at 13q12.12 are associated with high myopia in the Han Chinese population. Am. J. Hum. Genet. 88, 805-813 (2011).

37. Fan, Q. et al. Genetic variants on chromosome 1q41 influence ocular axial length and high myopia. PLoS Genet. 8, e1002753 (2012).

38. Hysi, P. G. et al. A genome-wide association study for myopia and refractive error identifies a susceptibility locus at 15q25. Nat. Genet. 42, 902-905 (2010)

39. Kiefer, A. K. et al. Genome-wide analysis points to roles for extracellular matrix remodeling, the visual cycle, and neuronal development in myopia. PLoS Genet 9, e1003299 (2013)

40. Verhoeven, V. J. et al. Large scale international replication and meta-analysis study confirms association of the 15q14 locus with myopia. The CREAM consortium. Hum. Genet. 131, 1467-1480 (2012).

41. Ashby, R., Kozulin, P., Megaw, P. L. \& Morgan, I. G. Alterations in ZENK and glucagon RNA transcript expression during increased ocular growth in chickens. Mol. Vis. 16, 639-649 (2010).

42. Deans, M. R., Volgyi, B., Goodenough, D. A., Bloomfield, S. A. \& Paul, D. L. Connexin36 is essential for transmission of rod-mediated visual signals in the mammalian retina. Neuron 36, 703-712 (2002).

43. Lee, E. J. et al. The immunocytochemical localization of connexin 36 at rod and cone gap junctions in the guinea pig retina. Eur. J. Neurosci. 18, 2925-2934 (2003).

44. Feigenspan, A. et al. Expression of connexin 36 in cone pedicles and OFF-cone bipolar cells of the mouse retina. J. Neurosci. 24, 3325-3334 (2004). 
45. Lin, B., Jakobs, T. C. \& Masland, R. H. Different functional types of bipolar cells use different gap-junctional proteins. J. Neurosci. 25, 6696-6701 (2005).

46. Han, Y. \& Massey, S. C. Electrical synapses in retinal ON cone bipolar cells: subtype-specific expression of connexins. Proc. Natl Acad. Sci. USA 102, 13313-13318 (2005).

47. Schubert, T. et al. Connexin 36 mediates gap junctional coupling of alphaganglion cells in mouse retina. J. Comp. Neurol. 485, 191-201 (2005).

48. Volgyi, B., Abrams, J., Paul, D. L. \& Bloomfield, S. A. Morphology and tracer coupling pattern of alpha ganglion cells in the mouse retina. J. Comp. Neurol. 492, 66-77 (2005).

49. Feigenspan, A., Teubner, B., Willecke, K. \& Weiler, R. Expression of neuronal connexin36 in AII amacrine cells of the mammalian retina. J. Neurosci. 21, 230-239 (2001)

50. Mills, S. L., O’Brien, J. J., Li, W., O’Brien, J. \& Massey, S. C. Rod pathways in the mammalian retina use connexin 36. J. Comp. Neurol. 436, 336-350 (2001).

51. Sohl, G., Joussen, A., Kociok, N. \& Willecke, K. Expression of connexin genes in the human retina. BMC Ophthalmol. 10, 27 (2010).

52. Pang, J. J., Paul, D. L. \& Wu, S. M. Survey on amacrine cells coupling to retrograde-identified ganglion cells in the mouse retina. Invest. Ophthalmol. Vis. Sci. 54, 5151-5162 (2013).

53. Abbott, C. J., Percival, K. A., Martin, P. R. \& Grunert, U. Amacrine and bipolar inputs to midget and parasol ganglion cells in marmoset retina. Vis. Neurosci. 29, 157-168 (2012).

54. Solomon, S. G., Martin, P. R., White, A. J., Ruttiger, L. \& Lee, B. B. Modulation sensitivity of ganglion cells in peripheral retina of macaque. Vision Res. 42, 2893-2898 (2002).

55. Calkins, D. J. \& Sterling, P. Microcircuitry for two types of achromatic ganglion cell in primate fovea. J. Neurosci. 27, 2646-2653 (2007).

56. Jacoby, R., Stafford, D., Kouyama, N. \& Marshak, D. Synaptic inputs to ON parasol ganglion cells in the primate retina. J. Neurosci. 16, 8041-8056 (1996).

57. Bordt, A. S., Hoshi, H., Yamada, E. S., Perryman-Stout, W. C. \& Marshak, D. W. Synaptic input to OFF parasol ganglion cells in macaque retina. J. Comp. Neurol. 498, 46-57 (2006).

58. Queiros, A., Gonzalez-Meijome, J. M., Jorge, J., Villa-Collar, C. \& Gutierrez, A. R. Peripheral refraction in myopic patients after orthokeratology. Optom. Vis. Sci. 87, 323-329 (2010).

59. Anstice, N. S. \& Phillips, J. R. Effect of dual-focus soft contact lens wear on axial myopia progression in children. Ophthalmology 118, 1152-1161 (2011).

60. Sankaridurg, P. et al. Decrease in rate of myopia progression with a contact lens designed to reduce relative peripheral hyperopia: one-year results. Invest. Ophthalmol. Vis. Sci. 52, 9362-9367 (2011).

61. Berntsen, D. A., Barr, C. D., Mutti, D. O. \& Zadnik, K. Peripheral defocus and myopia progression in myopic children randomly assigned to wear single vision and progressive addition lenses. Invest. Ophthalmol. Vis. Sci. 54, 5761-5770 (2013).

62. Kim, A. S., Anderson, S. A., Rubenstein, J. L., Lowenstein, D. H. \& Pleasure, S. J. Pax-6 regulates expression of SFRP-2 and Wnt-7b in the developing CNS. J. Neurosci. 21, RC132 (2001).
63. Zhang, J. et al. Isoform- and dose-sensitive feedback interactions between paired box 6 gene and delta-catenin in cell differentiation and death. Exp. Cell Res. 316, 1070-1081 (2010).

64. Liu, H., Mohamed, O., Dufort, D. \& Wallace, V. A. Characterization of Wnt signaling components and activation of the Wnt canonical pathway in the murine retina. Dev. Dyn. 227, 323-334 (2003).

65. Yang, I., Chang, O., Lu, Q. \& Kim, K. Delta-catenin affects the localization and stability of p120-catenin by competitively interacting with E-cadherin. Mol. Cells 29, 233-237 (2010).

66. Hao, H. X. et al. ZNRF3 promotes Wnt receptor turnover in an R-spondinsensitive manner. Nature 485, 195-200 (2012).

67. Nakata, I. et al. Association between the cholesteryl ester transfer protein gene and polypoidal choroidal vasculopathy. Invest. Ophthalmol. Vis. Sci. 54, 6068-6073 (2013).

68. Nakata, I. et al. Prevalence and characteristics of age-related macular degeneration in the Japanese population: the Nagahama study. Am. J. Ophthalmol. 156, 1002-1009.e2 (2013).

69. Li, S. et al. Flicker downregulates the content of crystallin proteins in formdeprived C57BL/6 mouse retina. Exp. Eye Res. 101, 1-8 (2012).

70. Zhou, X. et al. Experimental murine myopia induces collagen type Ialphal (COL1A1) DNA methylation and altered COL1A1 messenger RNA expression in sclera. Mol. Vis. 18, 1312-1324 (2012)

\section{Acknowledgements}

We thank Ms Kaori Misono, Ms Hatsue Hamanaka and Ms Miwa Fukami for their assistance in animal experiments, IHC and genotyping. We also thank Japan Eye Bank Association for its financial support.

\section{Author contributions}

M.M., K.Y., Y.T., P.N.B. and N.Y. designed the study. M.M., K.Y., K.S., S.M., H.N., I.N., Y.A.-K., N.G., A.T., S.K., M.M. and K.O.-M. gathered the clinical data. Y.T., C.-C.K., P.C., F.Q., A.M., O.P., C.H., A.F.W., H.C., A.J.R., M.S., M.T., D.A.M., A.W.H., G.C., Y.S. L.H., Z.Y., K.H.L., P.Y.P.K., M.K.H.Y, S.P.Y., N.M., S.M.G., V.V., T.A., S.-M.S., E.-S.T., T.Y.W. and C.-Y.C. generated the genetic data. M.M., P.C., F.Q., A.M. and R.Y. analysed the data. M.M., K.Y., Y.T., P.N.B. and R.Y. interpreted the data. M.M., K.Y., S.P.Y., V.V and P.N.B. drafted the paper. All the authors contributed towards revision of the paper.

\section{Additional information}

Supplementary Information accompanies this paper at http://www.nature.com/ naturecommunications.

Competing financial interests: The authors declare no competing financial interests.

Reprints and permission information is available online at http://npg.nature.com/ reprintsandpermissions.

How to cite this article: Miyake, M. et al. Identification of myopia-associated WNT7B polymorphisms provides insights into the mechanism underlying the development of myopia. Nat. Commun. 6:6689 doi: 10.1038/ncomms7689 (2015).

\section{Nagahama Study Group}

\section{Takeo Nakayama ${ }^{25}$, Akihiro Sekine ${ }^{26}$ and Shinji Kosugi ${ }^{27}$}

${ }^{25}$ Department of Health Informatics, Kyoto University School of Public Health, Kyoto, Japan. ${ }^{26}$ Department of Genome Informatics, Kyoto University School of Public Health, Kyoto, Japan. ${ }^{27}$ Department of Medical Ethics, Kyoto University School of Public Health, Kyoto, Japan. 